\title{
What is the Best Sampling Method to Monitor the Effect of Endoscopy Reprocessing?
}

\author{
Kwang Hyun Chung ${ }^{1}$ and Byung lk Jang ${ }^{2}$ \\ ${ }^{1}$ Department of Internal Medicine, Eulji Hospital, Eulji University School of Medicine, Seoul, ${ }^{2}$ Division of Gastroenterology and Hepatology, \\ Department of Internal Medicine, Yeungnam University College of Medicine, Daegu, Korea
}

See "Experimental Study to Develop a Method for Improving Sample Collection to Monitor Laryngoscopes after Reprocessing" by Savina Ditommaso, Monica Giacomuzzi, Elisa Ricciardi, et al., on page 463-469.

Multiple reports have described Carbapenem-resistant Enterobacteriaceae infection related with duodenoscope, and iatrogenic infections related with endoscopic procedures have been a major concern of endoscopy units. ${ }^{1-3}$ Therefore, the importance of an endoscopy reprocessing protocol has been emphasized, and the necessity of surveillance culture after reprocessing has also been raised. ${ }^{4}$ Recently, the US Food and Drug Administration, the Centers for Disease Control and Prevention (CDC), and the American Society for Microbiology, together with duodenoscope manufacturers and other experts, developed and distributed standardized protocols for duodenoscope surveillance sampling and culturing. ${ }^{5}$ In addition, in South Korea, a surveillance culture program is considered for inclusion as one of the evaluation criteria for acquiring a Certificate of Accredited Endoscopy Unit.

In retrograde surveillance of endoscopy reprocessing, sampling is a critical component; however, no standard methods or protocols have been established. Several studies have been conducted on surveillance culture of reprocessed endoscopes,

Received: August 28, 2018 Revised: September 11, 2018

Accepted: September 11, 2018

Correspondence: Byung Ik Jang

Division of Gastroenterology and Hepatology, Department of Internal Medicine, Yeungnam University College of Medicine, 170 Hyeonchung-ro, Nam-gu, Daegu 42415, Korea

Tel: +82-53-620-3831, Fax: +82-53-654-8386, E-mail: jbi@med.yu.ac.kr ORCID: https://orcid.org/0000-0002-3037-9272

(c) This is an Open Access article distributed under the terms of the Creative Commons Attribution Non-Commercial License (http://creativecommons.org/ licenses/by-nc/3.0) which permits unrestricted non-commercial use, distribution, and reproduction in any medium, provided the original work is properly cited. most of which obtained samples in accordance with the guideline of the European Society of Gastrointestinal Endoscopy and European Society of Gastroenterology and Endoscopy Nurses and Associates ${ }^{6}$ or $\mathrm{CDC}^{5}$, which consisted of flushing and/or brushing of working channels and swabs from the distal end, valve port, and elevator mechanism. However, data on the endoscope without channels or elevator mechanisms are scarce and concern is lacking on the necessity and significance of sampling at a relatively wide and smooth-surfaced area (which is expected to have fewer residual contaminants) such as the shaft of an endoscope.

The sampling method needs to be further defined and clarified. In this issue of Clinical Endoscopy, Ditommaso et al. published a study on the efficacy of a surface sampling device for surveillance culture after flexible fiberoptic laryngoscopes. ${ }^{7}$ In this study, the extraction efficiency of three different sampling devices (tissue non tissue [TNT] wipes, cellulose wipes, and hydrated sponge) inoculated with Bacillus atrophaeus spores were compared and TNT wipes showed the highest extraction efficiency (93\% vs. 52\% and 49\%, respectively). TNT wipes also showed high efficiency for both collection and extraction from the stainless-steel surface (87\%) and laryngoscope (85\%). TNT wipes showed a remarkable advantage to cellulose and hydrated sponge in terms of extraction efficiency, which can be used as a reference method.

Contaminants on the smooth surface of an endoscope may be easily removed with the routine endoscope reprocessing process, and most interest has been focused on the working channels or elevator mechanisms where the area contami- 
nants cannot be easily removed but are rather accumulated to form a biofilm. However, contamination of flexible fiberoptic laryngoscopes has been reported. ${ }^{8}$ Therefore, we must expand our attention to surface sampling and culturing during the surveillance for an endoscope reprocessing protocol. When high-yield sampling methods are applied, we could probably unravel knowledge we do not expect to find.

Meanwhile, many more issues need to be elucidated in surveillance sampling. Questions that need answers are as follows: How often should samples be collected? From where should the samples be collected? Are working channels and recesses enough, or should the handle and shaft of the endoscope be included, especially in scopes without channels? Do the sampling device and method really have a significant impact on the culture results? Should all positive culture results considered dangerous, or should they be considered to be dangerous only if they are at or above a cutoff or if they have some high-concern pathogen? What action should be taken after the danger signal is detected? High-level disinfection is not sterilization, and an endoscopic procedure is not an aseptic procedure; therefore, proper standards will ensure patient safety and prevent excessive effort, resources, and money from being wasted.

Surveillance sampling and culturing of endoscopes are a major concern to prevent iatrogenic infection in patients during endoscopic procedures. Surface sample culture of endoscopes was not demonstrated to be much better than channel flushing/brushing or endoscope tip swabbing, but an experimental study by Ditommaso et al. called attention to surface sample culture and showed the superiority of the TNT wipes in terms of extraction rate. ${ }^{7}$ More research studies are needed, and appropriate standards should be prepared to apply this study results in clinical practice.

Conflicts of Interest

The authors have no financial conflicts of interest.

\section{REFERENCES}

1. Epstein L, Hunter JC, Arwady MA, et al. New Delhi metallo- $\beta$-lactamase-producing carbapenem-resistant Escherichia coli associated with exposure to duodenoscopes. JAMA 2014;312:1447-1455.

2. Kovaleva J, Peters FT, van der Mei HC, Degener JE. Transmission of infection by flexible gastrointestinal endoscopy and bronchoscopy. Clin Microbiol Rev 2013;26:231-254.

3. Wendorf KA, Kay M, Baliga C, et al. Endoscopic retrograde cholangiopancreatography-associated AmpC Escherichia coli outbreak. Infect Control Hosp Epidemiol 2015;36:634-642.

4. Buss AJ, Been MH, Borgers RP, et al. Endoscope disinfection and its pitfalls--requirement for retrograde surveillance cultures. Endoscopy 2008;40:327-332.

5. FDA/CDC/ASM Working Group on Duodenoscope Culturing. Duodenoscope surveillance sampling \& culturing: reducing the risks of infection [Internet]. Atlanta (GA): Centers for Disease Control and Prevention; c2015 [updated 2018 Feb 23; cited 2018 Sep]. Available from: https://www.cdc.gov/hai/organisms/cre/cre-duodenoscope-surveillance-protocol.html.

6. Beilenhoff U, Neumann CS, Rey JF, Biering H, Blum R, Schmidt V. ESGE-ESGENA guideline for quality assurance in reprocessing: microbiological surveillance testing in endoscopy. Endoscopy 2007;39:175181.

7. Ditommaso S, Giacomuzzi M, Ricciardi E, Zotti C. Experimental study to develop a method for improving sample collection to monitor laryngoscopes after reprocessing. Clin Endosc 2018;51:463-469.

8. Muscarella LF. Prevention of disease transmission during flexible laryngoscopy. Am J Infect Control 2007;35:536-544. 